\title{
Axionlike particle inflation and dark matter
}

\author{
Wei Cheng $\odot,{ }^{1,2}$ Ligong Bian, ${ }^{3,4, *}$ and Yu-Feng Zhou ${ }^{2,5,6, \dagger}$ \\ ${ }^{1}$ School of Science, Chongqing University of Posts and Telecommunications, Chongqing 400065, \\ People's Republic of China \\ ${ }^{2}$ CAS Key Laboratory of Theoretical PHYSICS, Institute of Theoretical Physics, \\ Chinese Academy of Sciences, Beijing 100190, China and University of Chinese Academy of Sciences, \\ Beijing 100190, China \\ ${ }^{3}$ Department of Physics, Chongqing University, Chongqing 401331, China \\ ${ }^{4}$ Chongqing Key Laboratory for Strongly Coupled Physics, Chongqing 401331, China \\ ${ }^{5}$ School of Fundamental Physics and Mathematical Sciences, Hangzhou Institute for Advanced Study, \\ UCAS, Hangzhou 310024, China \\ ${ }^{6}$ International Centre for Theoretical Physics Asia-Pacific, Beijing/Hangzhou, China
}

(Received 21 April 2021; accepted 5 August 2021; published 7 September 2021)

\begin{abstract}
In this paper, we propose a generalized natural inflation model to study axionlike particle (ALP) inflation and dark matter. The model contains two additional parameters $\left(n_{1}, n_{2}\right)$ in comparison with the natural inflation model. The $n_{1}$ builds the connection with other ALP inflation models, and the $n_{2}$ controls the inflaton mass. After considering the cosmic microwave background and other cosmological observation limits, the model can realize small-field inflation with a wide mass range, and the axionlike particle inflaton considered here can serve as the dark matter candidate for certain parameter spaces.
\end{abstract}

DOI: 10.1103/PhysRevD.104.063010

\section{INTRODUCTION}

In cosmology, inflation has been firmly established by the accurate cosmic microwave background (CMB) measurement about the temperature and polarization anisotropy [1-3], and the existence of dark matter (DM) also has been confirmed by numerous cosmological observations [4-7], which lead a huge number of studies to describe inflation and DM at the same time [8-19]. The important ingredient of connecting inflation and DM is a reheating process. Usually, the functions of inflaton are to drive the inflation and reheat the Universe, while a new particle is introduced to act as the DM. To avoid introducing new particles, this paper extends the natural inflation (NI) to realize an axionlike particle (ALP) inflation; then, the vast majority of inflationary energy is converted into radiation energy, and the remaining inflaton acts as DM, to achieve the ALP serving as both inflaton and DM while reheating the Universe $[15,16]$.

The NI was first proposed in Ref. [20] to solve the hierarchy problem of inflation. Its appendant property, shift

\footnotetext{
*1gbycl@cqu.edu.cn

yfzhou@itp.ac.cn
}

Published by the American Physical Society under the terms of the Creative Commons Attribution 4.0 International license. Further distribution of this work must maintain attribution to the author(s) and the published article's title, journal citation, and DOI. Funded by SCOAP ${ }^{3}$. symmetry, allows an ALP to act as the inflaton. Besides, it keeps the flatness of potential energy, which is essential for driving the expansion of the Universe and generating density perturbations. The NI potential can be written as follows:

$$
V(\phi)=\Lambda^{4}\left[1+\cos \left(\frac{\phi}{f}\right)\right],
$$

where $\Lambda$ and $f$ are the inflaton energy density and decay constant, respectively. However, the model requires a super-Planckian decay constant, which may not be justified in the framework of quantum gravity, while the feasible parameter space of this simple NI is still shrinking according to the latest CMB data [21]. Thus, it has inspired many extension versions for the NI potential, such as the multi-NI model $[22,23]$ and other generalizations of the NI model [24], etc.

As a new attempt, we construct a generalized natural inflation (GNI) model to realize a single ALP natural inflation. In GNI, the new parameter $\epsilon$ is proportional to the decay constant $f$; thus, a large (small) $\epsilon$ may lead to a large (small)-field inflation. The $\epsilon$ is also proportional to the ALP mass, which will enable the ALP mass ranges from sub-eV to super-GeV. Moreover, GNI can be reduced to the previous models by fixing some parameters, which are elaborated in the main body.

After the inflation finished, the Universe was stretched and diluted extremely; thus, the temperature of the 
Universe became very low so that a reheating process is required to reheat the Universe $[25,26]$. Many models are devoted to describing this process, such as perturbation reheating [27-30], nonperturbation reheating [31,32], and instant reheating $[33,34]$. In this paper, the reheating of the Universe is implemented by the ALP inflaton decay. It is worth noting that for large mass ALP inflaton, it will decay quickly and cannot serve as the DM, but for small mass, ALP inflaton can be a long-lived DM. However, the Universe cannot be directly reheated using the usual methods. In order to solve this problem, we find that the inflation field is a damped oscillation motion near the minimal potential energy according to its equation of motion. That is, the inflationary field begins to oscillate at a certain time after the inflation finished, and this oscillation is the damped oscillation of amplitude attenuation. When the mass in the ALP decay rate is an effective mass that is related to the oscillation amplitude $[15,16]$, the decay rate at the beginning of the oscillation may be very large, and then decreases rapidly, which not only solves the problem of reheating the Universe with ALP inflaton, but also makes it possible for ALP inflaton to act as long-lived DM at the same time [15-19,35,36].

This work is organized as follows. In Sec. II, we introduce the GNI model, In Sec. II B, we explore the ingredients of the slow-roll inflation and the inflaton mass analytically in the GNI, and we analyze the suppression effect of the ALP mass. In Sec. III, we calculate the evolution of energy density in the reheating phase and further study the ALP DM relic density. Finally, we briefly summarize in Sec. IV.

\section{THE MODEL}

In this section, we elaborate on the inflationary potential of the GNI model, the corresponding ALP inflation, and the ALP mass.

\section{A. Inflationary potential}

We extend the NI potential Eq. (1) as follows:

$$
\begin{aligned}
V(\phi)= & \Lambda^{4}\left[\cos \left(\frac{\phi}{f}\right)+\epsilon \cos \left(\frac{\phi}{f}\right)\right. \\
& \left.+\exp \left[\frac{1}{n_{1} !} \cos \left(\frac{\phi}{f}+\frac{\pi}{n_{1} !}\right)^{n_{1}}\right]\right]^{n_{2}}+C,
\end{aligned}
$$

where the $C$ is a constant that shifts the minimum of the potential to zero, the term $\epsilon \cos \left(\frac{\phi}{f}\right)$ is the extension for the $\cos \left(\frac{\phi}{f}\right)$ term with the value of $\epsilon$ closing to 0 , and the new parameter $\epsilon$ will give the mass of ALP, which also distinguishes our model from the multi-NI model $[15,16,22,23]$. In addition, the positiveness of inflaton mass leads to $\epsilon>0$. As is shown later, $n_{2}$ can greatly magnify or shrink the mass and is highly related with the
DM property. The exponential term, a slight change for the number 1 in Eq. (1), ensures our model can produce very little ALP mass after considering the confines from CMB, which makes it possible for ALP to act as both inflaton and ALP DM while ALP reheats the Universe. In comparison with other extended NI models, the positive integers $n_{1}$ and $n_{2}$ make GNI more universal. The GNI will reduce to other generalizations of the NI model [24] when $n_{1}=0$, and it will further reduce to the NI model when $n_{1}=0$ and $n_{2}=1$. Moreover, the parameter $n_{1}$ can be fixed as 1 or 2 by considering the following analysis about the shape of the inflationary potential.

The GNI model is a slight extension of the NI model, and $\phi=0$ and $\phi=\pi f$ are still the maximum and minimum points of the inflationary potential, respectively, yielding

$$
\begin{gathered}
\left.V^{\prime}(\phi)\right|_{\phi=0}=0,\left.\quad V^{\prime}(\phi)\right|_{\phi=\pi f}=0, \\
\left.V^{\prime \prime}(\phi)\right|_{\phi=0}<0,\left.\quad V^{\prime \prime}(\phi)\right|_{\phi=\pi f}>0,
\end{gathered}
$$

where the prime denotes the derivative with respect to $\phi$. For convenience, the analytical expressions of the first and second derivatives of the potential are shown in the Appendix.

The first condition in Eq. (3) leads to $n_{1}=0,1$, and 2. For $n_{1}=0$, GNI will reduce to other generalizations of the NI model [24], and we skip the discussion of this scenario. The $n_{1}=1$ scenario is excluded by the second condition in Eq. (4), and the last scenario $n_{1}=2$ satisfies all of the conditions given in Eq. (4). For $n_{1}=2,\left.V^{\prime \prime}(\phi)\right|_{\phi=0}<0$ and $\left.V^{\prime \prime}(\phi)\right|_{\phi=\pi f}>0$ require that $\epsilon>0$, and $n_{2}$ should be an odd number. Furthermore, two inflection points around the $\phi=\pi f$ points can be obtained in terms of the first derivatives of the potential. This also can be seen from the top panel of Fig. 1.

GNI can produce not only the small-field inflation that is shown obviously in the bottom panel of Fig. 1 but also the large-field inflation. Since the large-field inflation has a similar shape to the small-field potential, we do not show it here. ALP can achieve the unification of ALP inflaton and ALP DM for the small-field inflation, but not for the largefield inflation due to the large mass. The large-field inflation will destroy the flatness of the potential and generate a gravitational wave [37], while the small-field inflation is reversed. For the study of the tensor-to-scalar ratio $r$ for large- and small-field inflations, see the next subsection.

\section{B. ALP inflation}

We briefly review the slow-roll inflation in this subsection. During the inflation, the slow-roll inflation has two characteristics: The kinetic energy of inflaton is much less than that of the potential energy, and the change rate of kinetic energy is much less than that of the cosmic 

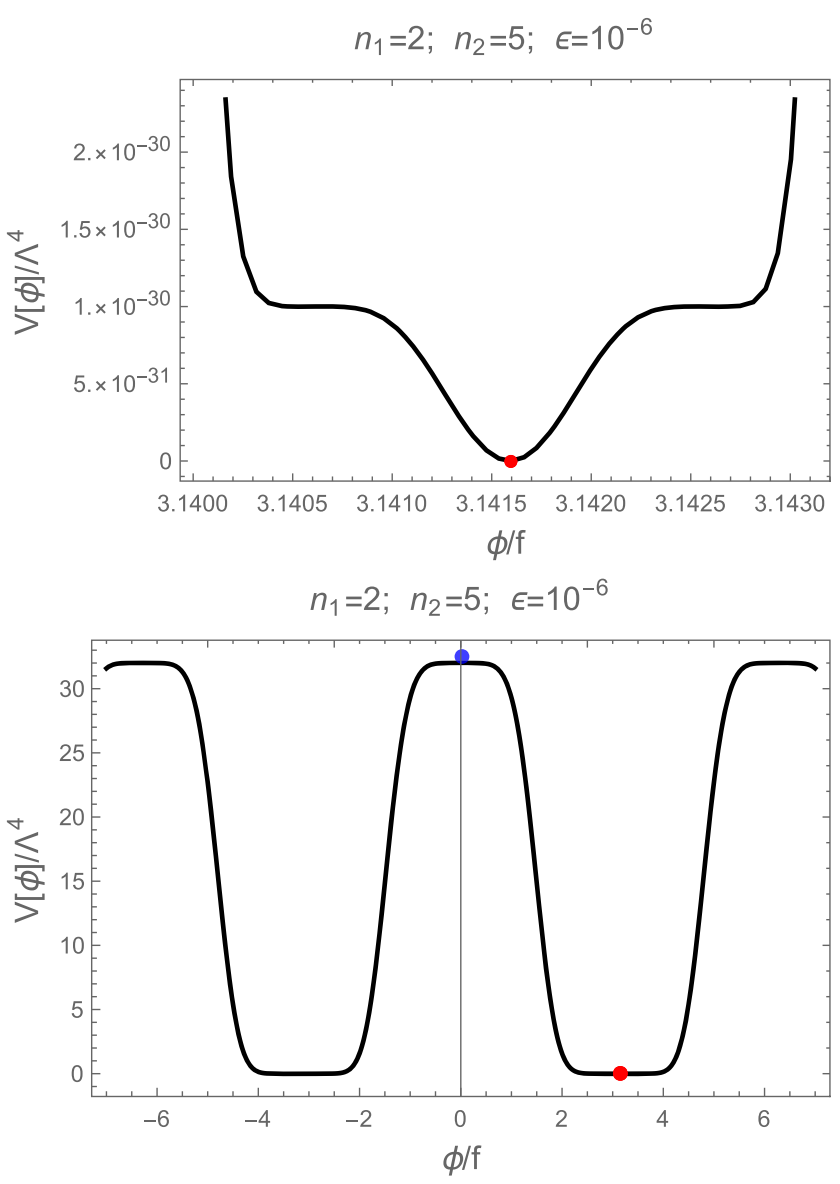

FIG. 1. Small-field inflation potential shape for the GNI. (Top) Corresponding true vacuum points of the small-field inflation potential. (Bottom) The small-field inflation potential. The red points represent the true vacuum points, and slow-roll inflation is possible around the blue dot.

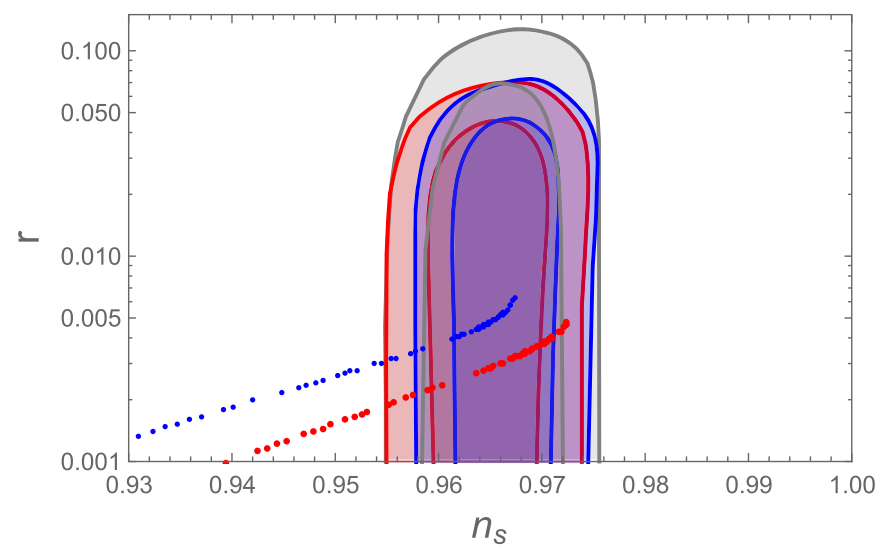

expansion, which are quantitatively described by the two inequalities $\varepsilon(\phi) \ll 1$, and $\eta(\phi) \ll 1$, where the slow-roll parameters are defined as

$\varepsilon(\phi) \equiv \frac{M_{\mathrm{pl}}^{2}}{2}\left(\frac{V^{\prime}(\phi)}{V(\phi)}\right)^{2}, \quad \eta(\phi) \equiv M_{\mathrm{pl}}^{2}\left(\frac{V^{\prime \prime}(\phi)}{V(\phi)}\right)$,

where the prime denotes the derivative with respect to $\phi$.

If anyone of the conditions breaks, i.e., $\varepsilon=1$ or $|\eta|=1$, the inflation will finish. This can be used to set the field value $\left(\phi_{\text {end }}\right)$ at the end of inflation.

The e-folding number $N_{I}$ is written as

$$
N_{I}=\int H d t
$$

This will give the initial value $\phi_{I}$ for a fixed e-folding number. We take $N_{I}=60$ to conduct our calculations.

Up to the first order level, the scalar spectral index $n_{s}$ and the tensor-to-scalar ratio $r$ can be expressed as

$$
n_{s}=1-6 \varepsilon+2 \eta
$$

and

$$
r=16 \varepsilon .
$$

Here, $n_{s}$ is dominated by the shape of the inflationary potential, and $r$ is associated with the inflationary energy scale [22].

Since the calculation of $r, n_{s}$ is related to the model potential, the value of $r, n_{s}$ will include the model parameters $\epsilon, n_{1}, n_{2}, f, \Lambda$, and the e-folding number $N$. We first fix $n_{1}=2$ and eliminate the parameter $\Lambda$ by using the confine of the scalar fluctuation amplitude from CMB. We further fix $\epsilon, n_{2}$, and $N$, and we then show the blue/red

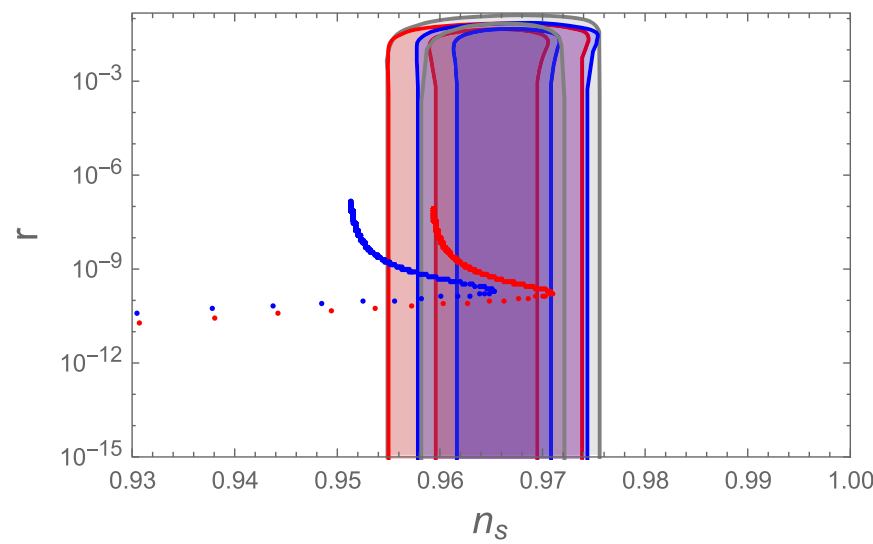

FIG. 2. (Left) Large-field inflation predictions in $\left(n_{s}, r\right)$ planes with $\epsilon=10^{-1}, n_{2}=5$. (Right) Small-field inflation predictions in $\left(n_{s}, r\right)$ planes with $\epsilon=10^{-6}, n_{2}=5$. Red (blue) points can be obtained by changing $f$; its corresponding e-folding number is $N=60$ $(N=50)$. Gray, red, and blue shadows stand for TT, TE, EE + lowE + lensing, TT, TE, EE + lowE + lensing $+\mathrm{BK} 15$, and $\mathrm{TT}, \mathrm{TE}, \mathrm{EE}+$ lowE + lensing + BK15 + BAO constraints [21]. 
points in the $\left(r-n_{s}\right)$ plane in Fig. 2 by changing $f$. We find that both large- and small-field inflations can be realized for $n_{2}=5$. The small value of $\epsilon$ corresponds to the small decay constant $f$ and then leads to small-field inflation. The largefield inflation may generate sizable tensor perturbations, which further lead to sizable gravity waves that are beyond the scope of this work.

The tensor-to-scalar ratio $r$, as well as the scalar spectral index $n_{s}$, are tightly constrained by the Planck data combined with CMB observations as [21]

$$
\begin{aligned}
& n_{s}=0.9649 \pm 0.0042, \\
& r<0.10 \quad(95 \% \mathrm{CL})
\end{aligned}
$$

There is another important experimental observation, i.e., amplitude of scalar fluctuations $\Delta_{\mathcal{R}}$,

$$
\Delta_{\mathcal{R}}=\frac{1}{24 \pi^{2} M_{\mathrm{pl}}^{4}} \frac{V(\phi)}{\varepsilon(\phi)},
$$

where $\Delta_{\mathcal{R}} \simeq e^{3.098} \times 10^{-10} \simeq 2.2 \times 10^{-9}$ [1], which will limit the height of the potential. For a usual extended NI, this bound will eliminate the suppression effect from the preparameter of the mass and further lead to a large mass, which makes it impossible for the inflaton to serve as the DM, while the GNI can solve this problem well.

To investigate the inflaton mass, we need to investigate the second derivative of potential at the true vacuum, i.e., minimal point of potential $(\phi=\pi f)$. The inflaton mass of large-/small-field inflation have the same form as follows:

$$
m_{\phi}=n_{2}^{1 / 2}(2+\epsilon)^{1 / 2} \epsilon^{\left(n_{2}-1\right) / 2} \frac{\Lambda^{2}}{f} .
$$

This indicates that $\epsilon$ will give the ALP mass, and the mass will decrease rapidly with the increase of $n_{2}$ due to the factor $\epsilon^{\left(n_{2}-1\right) / 2}$, which makes a wide range of ALP mass.

We plot the decay constant $f$ as a function of the ALP mass and $\epsilon$ for several values of $n_{2}$ with $n_{1}=2$ in Fig. 3, in which all shadows are confined by CMB data. For a fixed $n_{2}$, the decay constant $f$ increases monotonically with the parameter $\epsilon$. The reason for not considering the lower $\epsilon / f$ values is that the lower $\epsilon / f$ values correspond to the lower ALP mass, and smaller mass regions are not necessary to consider for practical purposes. With the increasing of $n_{2}$, the decay constant $f$ and the parameter $\epsilon$ remain almost unchanged, while with the mass falling fast an extremely small inflaton mass can also be obtained. The reason is that there is a suppression effect for the mass from the power exponent $\left(n_{2}\right)$, i.e., mass $\sim \epsilon^{\left(n_{2}-1\right) / 2}$ with $0<\epsilon<1$ in terms of Eq. (12).

Note that we treat ALP as both inflaton and DM in the following section, and ALP should have a small mass. However, the ALP mass has a large value for the large-field inflation, we do not study this scenario in the following section.

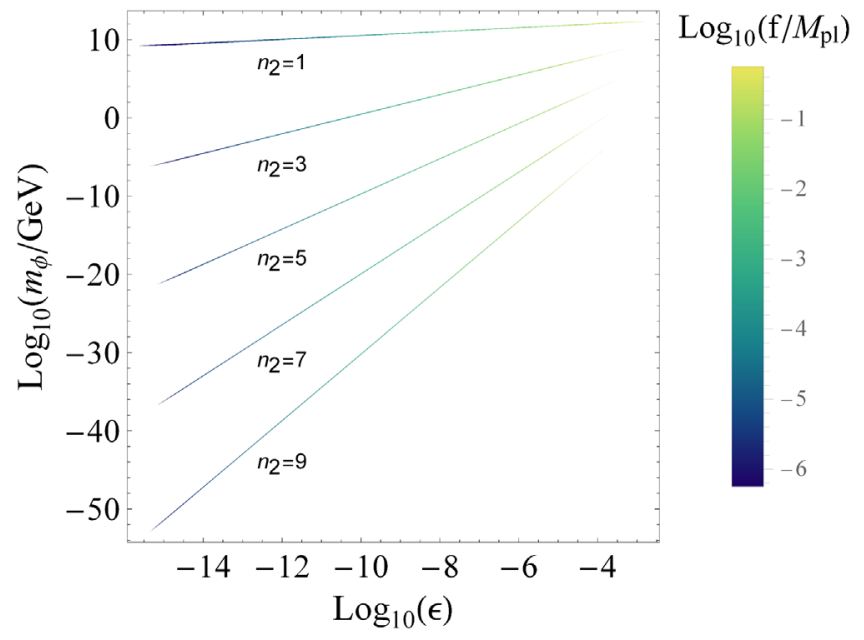

FIG. 3. The ALP mass $m_{\phi}$ as a function of parameter $\epsilon$ for several values of $n_{2}$ with $n_{1}=2$. All the shades are the feasible regions under the latest $\mathrm{CMB}$ data constraints.

\section{DARK MATTER}

In this paper, we only consider ALP inflaton coupled to photons; the Lagrangian can be written as [38]

$$
\mathcal{L}_{\mathrm{ALP}}=\frac{1}{2} \partial_{\mu} \phi \partial^{\mu} \phi-\frac{1}{2} m_{\phi}^{2} \phi^{2}-\frac{1}{4} g_{\phi \gamma \gamma} \phi F_{\mu \nu} \tilde{F}^{\mu \nu}
$$

where $\tilde{F}^{\mu \nu}$ is the dual electromagnetic field-strength tensor and the coupling $g_{\phi \gamma \gamma}=c_{r} \alpha /(2 \pi f)$ with the ALP decay constant $f$. We restrict the parameter $c_{r}$ in the range of $10^{-3}-10^{2}$ to do the numerical analysis. The perturbative decay rate can be obtained as follows:

$$
\Gamma_{0}(\phi \rightarrow \gamma \gamma)=\frac{\alpha^{2} c_{r}^{2}}{64 \pi^{3}} \frac{m_{\phi}^{3}}{f^{2}}
$$

The reheating epoch is a process that follows inflation, and the motion of the field in this epoch is actually a damped oscillation. Specifically, the equation of motion for the ALP zero mode in the early Universe is

$$
\ddot{\phi}+3 H \dot{\phi}+m_{\phi}^{2} \phi=0
$$

where the Hubble parameter $H^{2}=8 \pi \rho_{\text {tot }} / 3 M_{p}^{2}$. When the Hubble parameter becomes comparable to $m_{\phi}$, i.e., $m_{\phi}=q H$ with $q \simeq 3$, the field begins to oscillate with initial energy density $\rho_{\phi}=\frac{1}{2} m_{\phi}^{2} f^{2} \theta_{0}^{2}$, which will affect the DM relic abundance today [38]. ${ }^{1}$ The solution for the above field equation of motion can be written as the following oscillation form [35]

\footnotetext{
${ }^{1}$ Here, $\theta_{0}$ is the initial misalignment angle, usually $\theta_{0} \sim \mathcal{O}(1)$.
} 


$$
\phi(t)=\frac{\Phi_{0} M_{\mathrm{pl}}}{m_{\phi} t} \sin \left(m_{\phi} t\right) .
$$

The initial oscillation energy also can be approximately expressed as $\frac{1}{2} m_{\phi}^{4} \phi_{0}^{2}$. Thus, we can relate $\phi_{0}$ to $\theta_{0}$ by equating the two kinds of initial oscillation energy, i.e., $\frac{1}{2} m_{\phi}^{2} f^{2} \theta_{0}^{2}=\frac{1}{2} m_{\phi}^{4} \phi_{0}^{2}$.

We can obtain the effective ALP decay width in the reheating epoch as follows: as the mass is the second derivative of the potential, and the corresponding field is going to oscillate around the true vacuum in the reheating phase, we assume that the ALP mass is an effective mass that is related to the amplitude of the inflationary potential, i.e., $m_{\text {eff }}^{2}=V^{\prime \prime}\left(\phi_{\text {amp }}\right)$. The effective decay rate can be obtained as $\Gamma_{\text {eff }}(\phi \rightarrow \gamma \gamma)$ by replacing the mass $m_{\phi}$ in the decay rate $\Gamma_{0}(\phi \rightarrow \gamma \gamma)$ with $m_{\text {eff }}$. From the top panel of Fig. 4, we can find that the effective decay width is large at the beginning of the field oscillation and then rapidly drops to a very low level, which provides the possibility for the inflaton to reheat the Universe and then decouple as a longlived DM.

After determining the effect decay width, we can use Boltzmann equations to study the evolution of energy density during reheating, as follows:
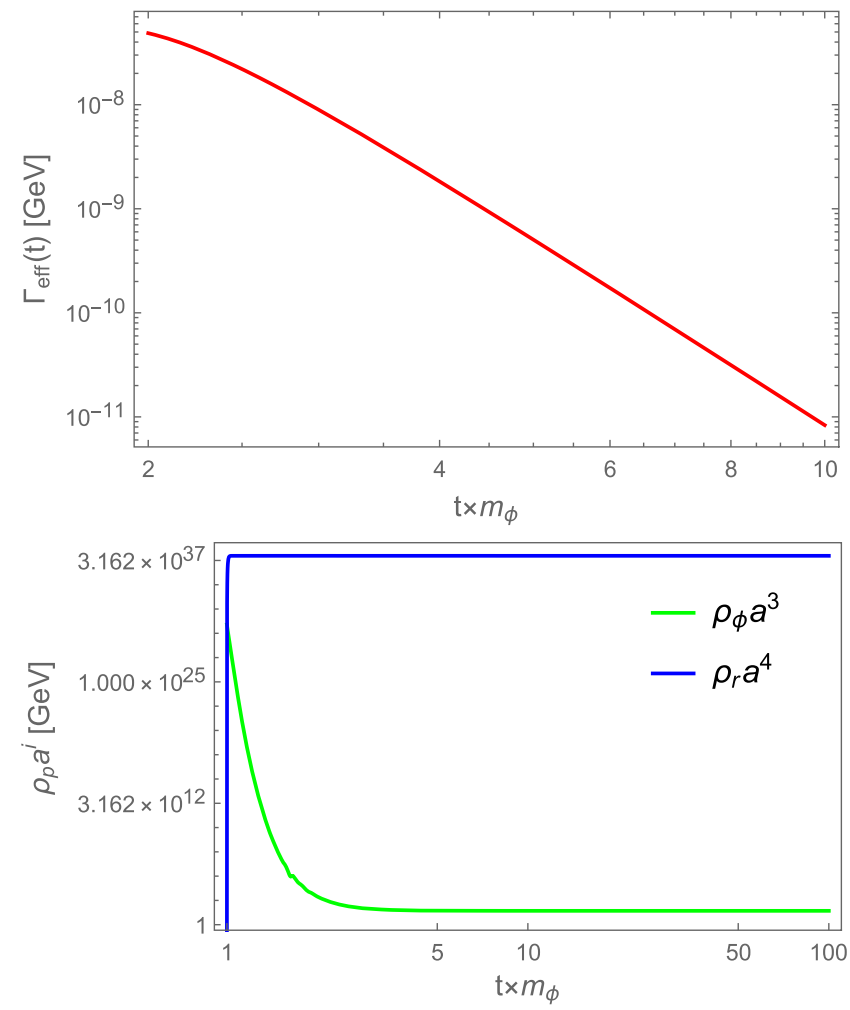

FIG. 4. (Top) The evolution of ALP decay width. (Bottom) The evolution of the comoving energy densities for the inflaton and radiation. The parameters are set as $\theta_{0}=0.95, n_{2}=5$, $c_{r} \approx 0.1356$, and $f=1.0476 \times 10^{15} \mathrm{GeV}$.

$$
\begin{aligned}
\dot{\rho}_{\phi}+3 H \rho_{\phi} & =-\Gamma_{\text {eff }} \rho_{\phi}, \\
\dot{\rho}_{r}+4 H \rho_{r} & =\Gamma_{\text {eff }} \rho_{\phi},
\end{aligned}
$$

where $\rho_{\phi}$ and $\rho_{r}$ denote the energy density of the inflaton and radiation, respectively. As the ALP potential is dominated by the quadratic term near the potential minimum, the ALP energy density $\rho_{\phi}$ may redshift as matter; thus, the coefficient of the first line of Eq. (17) should be 3 .

With the previous settings, we can solve the Boltzmann equations to get the energy evolution information, which is shown in the bottom panel of Fig. 4. We find that almost all the inflaton energy is immediately converted to the radiation because the decay rate $\Gamma_{\text {eff }}$ is much larger than $H$ at the beginning of the oscillation, which means instantaneous reheating takes place, and then, both the inflaton and the radiation energy density with corresponding scale factors remain stable because the decay rate $\Gamma_{\text {eff }}$ goes down fast and then close to 0 , which can be clearly seen from the top panel of Fig. 4. Consequently, the instantaneous heating of the Universe is achieved through ALP, and the ALP may decouple as a DM.

In the standard cosmology, the Universe will expand adiabatically after the end of reheating, and then, the ratio of the ALP number density to entropy density $n_{\phi} / s$ is conserved. Therefore, the ALP DM relic density today can be expressed as [38]

$$
\Omega_{\mathrm{DM}}=\frac{\rho_{\phi}\left(t_{\mathrm{RH}}\right)}{\rho_{c}}\left(\frac{T_{0}}{T_{\mathrm{RH}}}\right)^{3} \frac{g_{* S}\left(T_{0}\right)}{g_{* S}\left(T_{\mathrm{RH}}\right)},
$$

where $\rho_{c} \approx 10^{-5} h^{2} \mathrm{GeV} / \mathrm{cm}^{3}$ with $h \approx 0.68 \quad$ [39], $T_{0} \approx 2.7 \mathrm{~K}$. The reheating temperature $T_{\mathrm{RH}}$ is defined as the temperature of the Universe when $H(t) \approx \Gamma_{\text {eff }}(t)$ [40]. Considering $\rho_{r}=\left(\pi^{2} / 30\right) g(T) T^{4}$, the reheating temperature in this case can be estimated as $T_{\mathrm{RH}} \sim 10^{2} \mathrm{GeV}$, which satisfies the constraint of the reheating temperature more than the big bang nucleosynthesis (BBN) temperature $T_{\mathrm{BBN}} \sim 10^{-2} \mathrm{GeV}[41]$.

After further considering the bound from the BBN temperature and DM relic density $\Omega_{\mathrm{DM}} h^{2}=0.12$ [42], we plot the feasible points with $n_{2}=3,5,7,9$ in Fig. 5 (green points), where each point corresponds to an exact value $c_{r}$ that falls in the range of $10^{-3}-10^{2}$. For example, the triangular red dot in Fig. 5 corresponds to $c_{r}=0.1356$, which is given in the caption of Fig. 4. In Fig. 5, the darker green color corresponds to the case of the bigger $n_{2}$. We find that there is no point for the $n_{2}=1$ case, because the larger mass of ALP will decay completely in the reheating epoch and cannot serve as the DM in that case. The case $n_{2}=3$ is ruled out completely by the cosmology constraints that include optical, EBL, $\chi_{\text {ion }}$, $x$-rays, and DM limits; more details can be found in Ref. [43]. The cases of $n_{2}=5,7,9$ survive for the GNI model. 


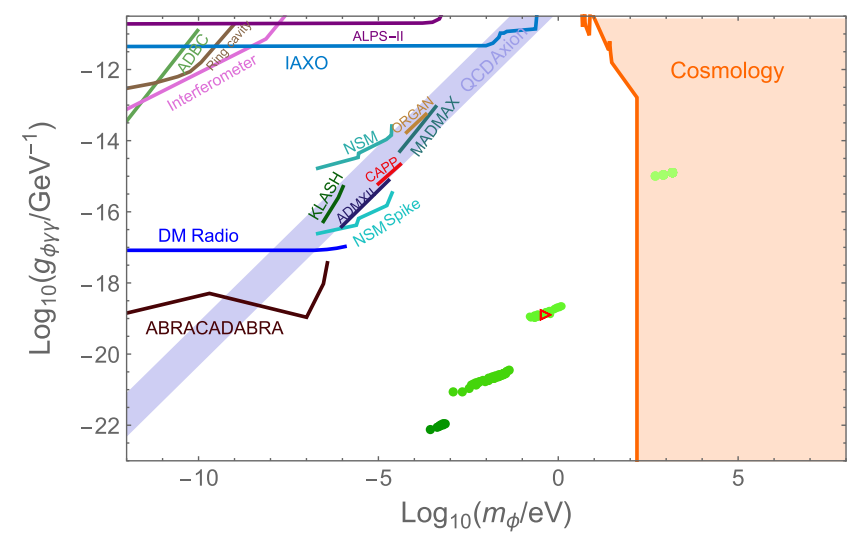

FIG. 5. The $m_{\phi}-g_{\phi \gamma \gamma}$ figure for $n_{2}=3,5,7,9$. The darker the green color is, the bigger the $n_{2}$ is. All the green points are bounded from the BBN temperature for the $T_{\mathrm{RH}}$ and DM relic density $\Omega h^{2}=0.12$. Those colored lines are the projected experimental reach [38]. The red triangle is the illustration point for ALP reheating and DM. Note that the case of $n_{2}=1$ is excluded.

\section{SUMMARY}

In this paper, we propose a GNI model with the parameters $f, \Lambda, n_{1}, n_{2}, \epsilon$, and $C$. We find that the parameter $n_{1}$ can only be equal to 0,2 due to the constraints of roll inflation and the true vacuum condition for potential, which bring the conditions of the maximal and minimal values for the potential. When the parameters $n_{1}, n_{2}$ take specific values, GNI can be simplified to some other models. Specifically, the GNI model can reduce to NI with $n_{1}=1, n_{2}=1$, and other generalizations of the natural inflation model with $n_{1}=1$. Besides, the GNI model with $n_{1}=2$ takes on unique characters. It not only contains the functions of other generalizations of the natural inflation model but also produces both large and small inflation, so we study it in depth.
We find that, for the scenario of $n_{1}=2$, the ALP mass is only sensitive to $n_{2}$ but not to the decay constant $f$ and parameter $\epsilon$, because the mass is proportional to $\epsilon^{\left(n_{2}-1\right) / 2}$ with $0<\epsilon<1$. Under the CMB data limits, the mass of ALP varies from super-GeV to sub-eV for $n_{2} \sim 1 \rightarrow 9$. ALP with a small mass is a long-lived particle and can serve as a DM. When inflation is finished, the ALP field will oscillate damped near the lowest potential, and the amplitude of the ALP field will decrease rapidly. Therefore, according to $\Gamma_{\text {eff }} \sim m_{\text {eff }}^{3}\left(\phi_{\text {amp }}\right)$, most of the ALP energy can be converted into radiation energy instantaneously to complete the heating of the Universe and leaving part of the energy as the longlived DM particle. After taking into account the CMB and other cosmological constraints, the GNI model can address both ALP inflation and DM for $n_{2}=5,7,9$.

\section{ACKNOWLEDGMENTS}

We thank Luca Visinelli for helpful discussions on ALP inflation and dark matter. This work was supported in part by the China Postdoctoral Science Foundation under Grants No. 2019TQ0329 and No. 2020M670476, the National Key R\&D Program of China under Grant No. 2017YFA0402204, the National Natural Science Foundation of China (NSFC) Grants No. 11825506, No. 11821505, No. U1738209, No. 11851303, and No. 11947302, the Natural Science Foundation of China under Grant No. 12047564, and the Fundamental Research Funds for the Central Universities under Grant No. 2020CDJQY-Z003. Ligong Bian was supported by the National Natural Science Foundation of China under Grants No. 12075041 and No. 12047564, and the Fundamental Research Funds for the Central Universities of China (Grants No. 2021CDJQY-011 and No. 2020CDJQY-Z003), and Chongqing Natural Science Foundation (Grants No. cstc2020jcyj-msxmX0814).

\section{APPENDIX: DERIVATIVES OF THE INFLATIONARY POTENTIAL}

The first and second derivatives of the potential can be written as follows:

$$
\begin{aligned}
\left.V^{\prime}(\phi)\right|_{\phi=0} & =-\frac{e^{\frac{\cos \left(\frac{\pi}{n_{1}} ! n_{1} n_{1}\right.}{n_{1} !}}\left(1+\epsilon+\exp ^{\frac{\left.\cos \left(\frac{\pi}{n_{1}}\right)^{n_{1}}\right)^{!}}{n_{1}}}\right)^{-1+n_{2}} n_{1} n_{2} \Lambda^{4} \cos \left(\frac{\pi}{n_{1} !}\right)^{-1+n_{1}} \sin \left(\frac{\pi}{n_{1} !}\right)}{f n_{1} !} \\
\left.V^{\prime}(\phi)\right|_{\phi=\pi f} & =-\frac{e^{\frac{-\cos \left(\frac{\pi}{n_{1}} !^{n_{1}} !\right.}{n_{1} !}}\left(-1-\epsilon+\exp ^{\frac{-\cos \left(\frac{\pi}{n_{1}} ! n_{1}\right.}{n_{1} !}}\right)^{-1+n_{2}} n_{1} n_{2} \Lambda^{4}\left(-\cos \left(\frac{\pi}{n_{1} !}\right)\right)^{n_{1}} \tan \left(\frac{\pi}{n_{1} !}\right)}{f n_{1} !} . \\
\left.V^{\prime \prime}(\phi)\right|_{\phi=0} & =-\frac{\epsilon(2+\epsilon)^{-1+n_{2}} n_{2} \Lambda^{4}}{f^{2}}, \\
\left.V^{\prime \prime}(\phi)\right|_{\phi=\pi f} & =\frac{(-\epsilon)^{-1+n_{2}}(2+\epsilon) n_{2} \Lambda^{4}}{f^{2}} .
\end{aligned}
$$


[1] P. Ade et al. (Planck Collaboration), Planck 2015 results. XX. Constraints on inflation, Astron. Astrophys. 594, A20 (2016).

[2] K. T. Story et al., A measurement of the cosmic microwave background damping tail from the 2500-square-degree SPTSZ survey, Astrophys. J. 779, 86 (2013).

[3] Z. Hou et al., Constraints on cosmology from the cosmic microwave background power spectrum of the $25002 \mathrm{deg}$ SPT-SZ survey, Astrophys. J. 782, 74 (2014).

[4] G. Hinshaw et al. (WMAP Collaboration), Nine-year Wilkinson microwave anisotropy probe (WMAP) observations: Cosmological parameter results, Astrophys. J. Suppl. 208, 19 (2013).

[5] B. Anton G, P. Vahe, and L. Roger, Gravitational lens models of arcs in clusters, Astrophys. J. 1, 350 (1990).

[6] P. Sikivie, Axion cosmology, Lect. Notes Phys. 741, 19 (2008).

[7] G. Bertone, D. Hooper, and J. Silk, Particle dark matter: Evidence, candidates and constraints, Phys. Rep. 405, 279 (2005).

[8] A. R. Liddle and L. A. Urena-Lopez, Inflation, Dark Matter and Dark Energy in the String Landscape, Phys. Rev. Lett. 97, 161301 (2006).

[9] A. R. Liddle, C. Pahud, and L. Urena-Lopez, Triple unification of inflation, dark matter, and dark energy using a single field, Phys. Rev. D 77, 121301 (2008).

[10] R. N. Lerner and J. McDonald, Gauge singlet scalar as inflaton and thermal relic dark matter, Phys. Rev. D 80, 123507 (2009).

[11] J. De-Santiago and J. L. Cervantes-Cota, Generalizing a unified model of dark matter, dark energy, and inflation with non canonical kinetic term, Phys. Rev. D 83, 063502 (2011).

[12] K. Mukaida and K. Nakayama, Dissipative effects on reheating after inflation, J. Cosmol. Astropart. Phys. 03 (2013) 002.

[13] T. Tenkanen, Feebly interacting dark matter particle as the inflaton, J. High Energy Phys. 09 (2016) 049.

[14] M. Bastero-Gil, R. Cerezo, and J. G. Rosa, Inflaton dark matter from incomplete decay, Phys. Rev. D 93, 103531 (2016).

[15] R. Daido, F. Takahashi, and W. Yin, The ALP miracle: Unified inflaton and dark matter, J. Cosmol. Astropart. Phys. 05 (2017) 044.

[16] R. Daido, F. Takahashi, and W. Yin, The ALP miracle revisited, J. High Energy Phys. 02 (2018) 104.

[17] L. Kofman, A. D. Linde, and A. A. Starobinsky, Reheating After Inflation, Phys. Rev. Lett. 73, 3195 (1994).

[18] D. Hooper, G. Krnjaic, A. J. Long, and S. D. Mcdermott, Can the Inflaton Also Be a Weakly Interacting Massive Particle?, Phys. Rev. Lett. 122, 091802 (2019).

[19] L. Kofman, A. D. Linde, and A. A. Starobinsky, Towards the theory of reheating after inflation, Phys. Rev. D 56, 3258 (1997).

[20] F. C. Adams, J. Bond, K. Freese, J. A. Frieman, and A. V. Olinto, Natural inflation: Particle physics models, power law spectra for large scale structure, and constraints from COBE, Phys. Rev. D 47, 426 (1993).
[21] Y. Akrami et al. (Planck Collaboration), Planck 2018 results. X. Constraints on inflation, arXiv:1807.06211.

[22] M. Czerny and F. Takahashi, Multi-natural inflation, Phys. Lett. B 733, 241 (2014).

[23] F. Takahashi and W. Yin, ALP inflation and big bang on Earth, J. High Energy Phys. 07 (2019) 095.

[24] J. B. Munoz and M. Kamionkowski, Equation-of-state parameter for reheating, Phys. Rev. D 91, 043521 (2015).

[25] A. D. Linde, Absence of particle creation in a hot Friedmann universe, JETP Lett. 35, 492 (1982).

[26] A. Albrecht, P. J. Steinhardt, M. S. Turner, and F. Wilczek, Reheating an Inflationary Universe, Phys. Rev. Lett. 48, 1437 (1982).

[27] L. Abbott, E. Farhi, and M. B. Wise, Particle production in the new inflationary cosmology, Phys. Lett. 117B, 29 (1982).

[28] A. Dolgov and A. D. Linde, Baryon asymmetry in inflationary universe, Phys. Lett. 116B, 329 (1982).

[29] A. Dolgov, Field model with a dynamic cancellation of the cosmological constant, JETP Lett. 41, 345 (1985).

[30] J. L. Cook, E. Dimastrogiovanni, D. A. Easson, and L. M. Krauss, Reheating predictions in single field inflation, J. Cosmol. Astropart. Phys. 04 (2015) 047.

[31] R. Allahverdi, A. Ferrantelli, J. Garcia-Bellido, and A. Mazumdar, Non-perturbative production of matter and rapid thermalization after MSSM inflation, Phys. Rev. D 83, 123507 (2011).

[32] A. S. Koshelev, K. Sravan Kumar, and A. A. Starobinsky, $R^{2}$ inflation to probe non-perturbative quantum gravity, J. High Energy Phys. 03 (2018) 071.

[33] G. N. Felder, L. Kofman, and A. D. Linde, Instant preheating, Phys. Rev. D 59, 123523 (1999).

[34] A. Kurkela and G. D. Moore, Thermalization in weakly coupled nonabelian plasmas, J. High Energy Phys. 12 (2011) 044.

[35] V. H. Cardenas, Inflation, reheating and dark matter, Phys. Rev. D 75, 083512 (2007).

[36] S. M. Choi, Y. J. Kang, H. M. Lee, and K. Yamashita, Unitary inflaton as decaying dark matter, J. High Energy Phys. 05 (2019) 060.

[37] A. D. Linde, Chaotic inflation, Phys. Lett. 129B, 177 (1983).

[38] N. Blinov, M. J. Dolan, P. Draper, and J. Kozaczuk, Dark matter targets for axionlike particle searches, Phys. Rev. D 100, 015049 (2019).

[39] M. Tanabashi et al. (Particle Data Group), Review of particle physics, Phys. Rev. D 98, 030001 (2018).

[40] D. J. H. Chung, E. W. Kolb, and A. Riotto, Production of massive particles during reheating, Phys. Rev. D 60, 063504 (1999).

[41] G. Steigman, Primordial nucleosynthesis in the precision cosmology era, Annu. Rev. Nucl. Part. Sci. 57, 463 (2007).

[42] P. Ade et al. (Planck Collaboration), Planck 2015 results. XIII. Cosmological parameters, Astron. Astrophys. 594, A13 (2016).

[43] D. Cadamuro, Cosmological limits on axions and axion-like particles, arXiv:1210.3196. 